\title{
Correction to: Gpx4, Selenov, and Txnrd3 Are Three Most Testis-Abundant Selenogenes Resistant to Dietary Selenium Concentrations and Actively Expressed During Reproductive Ages in Rats
}

\author{
Aiping Liu ${ }^{1,2}$. Fengna $\mathrm{Li}^{1} \cdot$ Ping $\mathrm{Xu}^{1,3}$ - Yanmei Chen ${ }^{1,4} \cdot$ Xiongshun Liang $^{5} \cdot$ Shijie Zheng ${ }^{6} \cdot$ Huicui Meng ${ }^{1,7}$. \\ Yumei Zhu ${ }^{5}$. Junluan $\mathrm{Mo}^{5}$. Chunmei Gong ${ }^{5}$. Ji-Chang Zhou ${ }^{1,5,7}$
}

Published online: 1 March 2022

(c) Springer Science+Business Media, LLC, part of Springer Nature 2022

\section{Correction to: Biological Trace Element Research} https://doi.org/10.1007/s12011-022-03118-5

The original version of this article unfortunately contained a mistake. The correct Figures 1 and 5 are presented here.

The original article has been corrected.

Publisher's Note Springer Nature remains neutral with regard to jurisdictional claims in published maps and institutional affiliations.

The original article can be found online at https://doi.org/10.1007/ s12011-022-03118-5.

Ji-Chang Zhou

zhoujch8@mail.sysu.edu.cn

1 School of Public Health (Shenzhen), Shenzhen Campus of Sun Yat-Sen University, Shenzhen 518107, Guangdong, China

2 Nanjing Gulou District Center for Disease Control and Prevention, Nanjing 210000, Jiangsu, China

3 Shenzhen Health Development Research and Data Management Center, Shenzhen 518028, Guangdong, China

4 Guangzhou Center for Disease Control and Prevention, Guangzhou 510000, Guangdong, China

5 Shenzhen Center for Chronic Disease Control, Shenzhen 518020, Guangdong, China

6 Service Center for Public Health of Bao' an District, Shenzhen 518018, Guangdong, China

7 Guangdong Province Engineering Laboratory for Nutrition Translation, Guangzhou 510080, Guangdong, China 

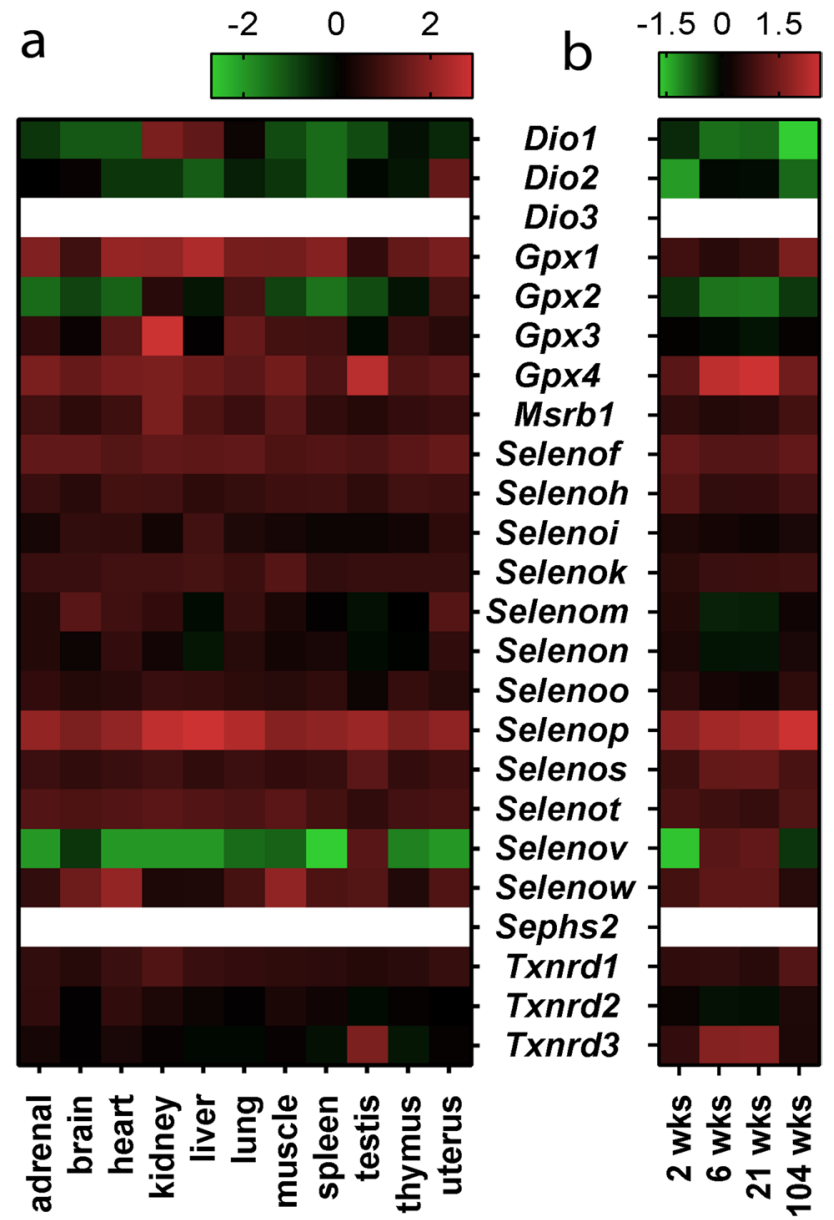

Fig. 1 mRNA abundances of rat selenogenome (except Dio3 and Sephs2) in various tissues at 21 weeks old (a) and in testis at 2, 6, 21, and 104 weeks (wks) old (b). Heatmaps were generated with the $1 \mathrm{~g}$ transformed means of reads per kilobase per million mapped reads of RNA-Seq data published in GenBank of NCBI, $n=4-8$ [15]
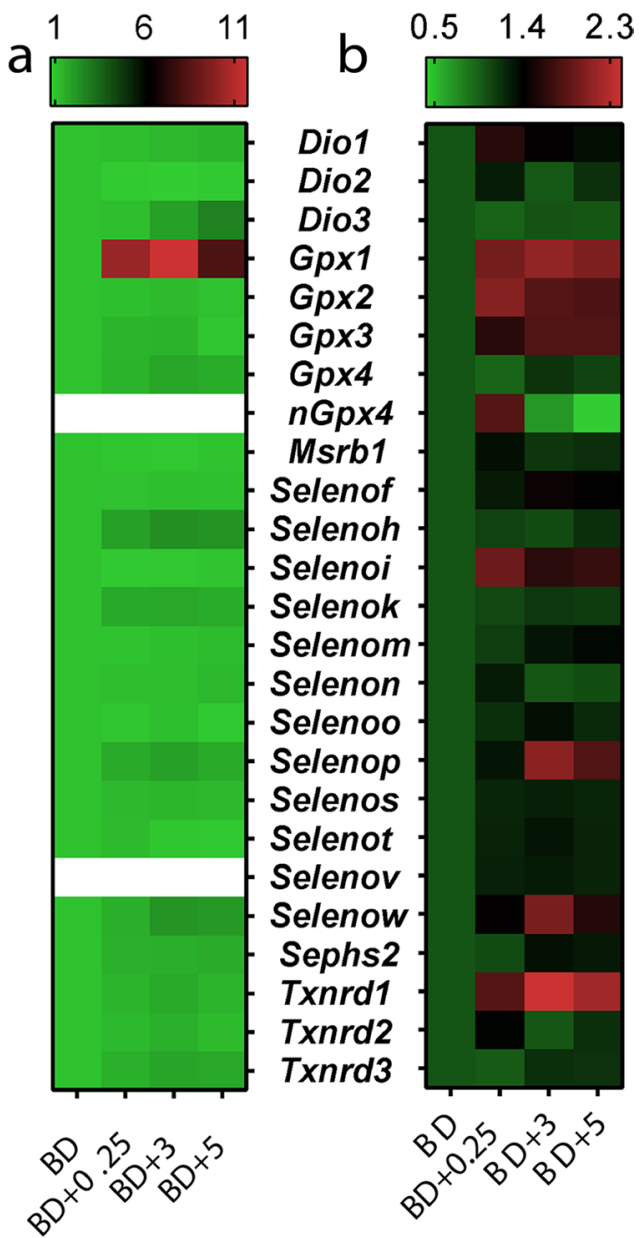

Fig. 5 mRNA abundances of rat selenogenome in the liver (a) and testis (b) of rats fed selenium (Se) deficient basal diet (BD) and BD plus Se $[\mathrm{BD}+0.25,3$, or $5(\mathrm{mg} \mathrm{Se} / \mathrm{kg})]$. Heatmaps were generated with the average fold changes of mRNA abundances against the BD group, and $n G p x 4$ and Selenov had no reliable data in the liver. $n=$ $8-12$ 